\title{
Danilo Silva
}

\section{Códigos de Permutação para Compressão de Dados e Modulação}

\section{DISSERTAÇÃO DE MESTRADO \\ DEPARTAMENTO DE ENGENHARIA ELÉTRICA \\ Programa de Pós-graduação em \\ Engenharia Elétrica}




\section{Pontifícia U Uiversidade Católica $_{\text {a }}$

Danilo Silva

Códigos de Permutação para Compressão de Dados e Modulação

Dissertação apresentada como requisito parcial para obtenção do grau de Mestre pelo Programa de Pós-graduação em Engenharia Elétrica do Departamento de Engenharia Elétrica da PUC-Rio

Orientador: Prof. Weiler Alves Finamore

Rio de Janeiro Março de 2005 


\section{Pontifícia U Uiversidade Católlica $_{\text {a }}$

Danilo Silva

\section{Códigos de Permutação para Compressão de Dados e Modulação}

Dissertação apresentada como requisito parcial para obtenção do grau de Mestre pelo Programa de Pósgraduação em Engenharia Elétrica do Departamento de Engenharia Elétrica do Centro Técnico Científico da PUCRio.Aprovada pela Comissão Examinadora abaixo assinada.

Prof. Weiler Alves Finamore

Orientador

Departamento de Engenharia Elétrica - PUC-Rio

Prof. Raimundo Sampaio Neto

Departamento de Engenharia Elétrica - PUC-Rio

Prof. Max Henrique Machado Costa

DECOM - Unicamp

Prof. José Eugênio Leal

Coordenador Setorial do Centro Técnico Científico -

PUC-Rio

Rio de Janeiro, 18 de Março de 2005 
Todos os direitos reservados. É proibida a reprodução total ou parcial do trabalho sem autorização da universidade, do autor e do orientador.

\section{Danilo Silva}

Graduou-se na Universidade Federal de Pernambuco, cursando Engenharia Elétrica, modalidade Eletrônica, no ano de 2002.

Ficha Catalográfica

Silva, Danilo

Códigos de Permutação para Compressão de Dados e Modulação/ Danilo Silva; orientador: Weiler Alves Finamore. - Rio de Janeiro : PUC-Rio, Departamento de Engenharia Elétrica, 2005.

v., 96 f.: il. ; $29,7 \mathrm{~cm}$

Dissertação (mestrado) - Pontifícia Universidade Católica do Rio de Janeiro, Departamento de Engenharia Elétrica.

Inclui referências bibliográficas.

1. Engenharia Elétrica - Teses. 2. Códigos de permutação. 3. Codificação de fonte. 4. Compressão com perdas. 5. Comunicação digital. 6. Modulação. 7. Teoria da informação. I. Finamore, Weiler Alves. II. Pontifícia Universidade Católica do Rio de Janeiro. Departamento de Engenharia Elétrica. III. Título. 


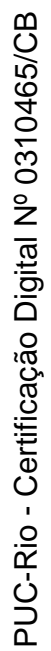

Para os meus pais, José e Neide. 


\section{Agradecimentos}

Em especial, aos meus pais, José Mercês Neto e Neide Maria da Silva, pelo apoio, carinho e dedicação imensuráveis, por acreditarem, investirem, e me fazerem superar todos os obstáculos, e à minha irmã Carolina Silva, por todo o carinho e apoio.

Ao meu orientador, prof. Weiler Finamore, não apenas por ter me fornecido uma excelente orientação, ao mesmo tempo competente, cuidadosa e motivante, mas também por ter sido um ótimo amigo, invariavelmente gentil, atencioso e compreensivo.

Aos meus professores do Departamento de Eletrônica e Sistemas da UFPE, em especial os profs. Ricardo Campello, Hélio Magalhães e Cecílio Pimentel, que contribuíram de forma decisiva para minha formação e minha motivação pela pesquisa e me forneceram os conselhos e a inspiração necessários para que eu trilhasse os caminhos que decidi seguir.

Aos professores Raimundo Sampaio e Max Costa, pela leitura cuidadosa e pelas valiosas sugestões para a melhoria deste trabalho.

Ao amigo Tiago Vinhoza, pela disposição quase irrestrita em me prestar sua competente e sem dúvida muito valiosa ajuda nos diversos momentos em que precisei ao longo deste trabalho.

A Amanda Cunha, por sua ajuda inestimável na preparação de figuras, revisão do texto e apresentação do trabalho, por suas sugestões sempre muito construtivas, e, principalmente, por todo o carinho, afeto, compreensão e apoio.

A todos os outros amigos que fiz no CETUC ao longo desse período, especialmente Fred, Marcelle, Aureo, Fabrício, Anna Carolina, Sérgio, Luís Eduardo e Fabian, e a todos os meus amigos de Recife, especialmente Marcos, Pablo, Elton, Yarly e Marcelle.

Agradeço também à toda a comunidade do CETUC e da PUC-Rio, pelo ótimo relacionamento, e ao $\mathrm{CNPq}$, pelo apoio financeiro. 


\section{Resumo}

Silva, Danilo; Finamore, Weiler Alves. Códigos de Permutação para Compressão de Dados e Modulação. Rio de Janeiro, 2005. 96p. Dissertação de Mestrado — Departamento de Engenharia Elétrica, Pontifícia Universidade Católica do Rio de Janeiro.

Códigos de permutação são uma interessante ferramenta matemática que pode ser empregada para construir tanto esquemas de compressão com perdas quanto esquemas de modulação em um sistema de transmissão digital. Códigos de permutação vetorial, uma extensão mais poderosa dos códigos de permutação escalar, foram recentemente introduzidos no contexto de compressão de fontes. Este trabalho apresenta novas contribuições a essa teoria e introduz os códigos de permutação vetorial no contexto de modulação. Para compressão de fontes, é demonstrado matematicamente que os códigos de permutação vetorial (VPC) têm desempenho assintótico idêntico ao do quantizador vetorial com restrição de entropia (ECVQ). Baseado neste desenvolvimento, é proposto um método eficiente para o projeto de VPC's. O bom desempenho dos códigos projetados com esse método é verificado através de resultados experimentais para as fontes uniforme e gaussiana: são exibidos VPC's cujo desempenho é semelhante ao do ECVQ e superior ao de sua versão escalar. Para o propósito de transmissão digital, é verificado que também a modulação baseada em códigos de permutação vetorial (VPM) possui desempenho superior ao de sua versão escalar. São desenvolvidas as expressões para o projeto ótimo de VPM, e um método é apresentado para detecção ótima de VPM em canais AWGN e com desvanecimento.

\section{Palavras-chave}

Códigos de permutação; codificação de fonte; compressão com perdas; comunicação digital; modulação; teoria da informação. 


\section{Abstract}

Silva, Danilo; Finamore, Weiler Alves. Permutation Codes for Data Compression and Modulation. Rio de Janeiro, 2005. 96p. MSc. Dissertation - Departamento de Engenharia Elétrica, Pontifícia Universidade Católica do Rio de Janeiro.

Permutation codes are an interesting mathematical tool which can be used to devise both lossy compression schemes and modulation schemes for digital transmission systems. Vector permutation codes, a more powerful extension of scalar permutation codes, were recently introduced for the purpose of source compression. This work presents new contributions to this theory and also introduces vector permutation codes for the purpose of modulation. For source compression, it is proved that vector permutation codes (VPC) have an asymptotical performance equal to that of an entropy-constrained vector quantizer (ECVQ). Based on this development, an efficient method is proposed for VPC design. Experimental results for Gaussian and uniform sources show that the codes designed by this method have indeed a good performance: VPC's are exhibited whose performances are similar to that of ECVQ and superior to those of their scalar counterparts. In the context of digital transmission, it is verified that also vector permutation modulation (VPM) is superior in performance to scalar permutation modulation. Expressions are developed for the optimal design of VPM, and a method is presented for maximum-likelihood detection of VPM in AWGN and fading channels.

\section{Keywords}

Permutation codes; source coding; lossy compression; communications theory; modulation; information theory. 


\section{Conteúdo}

1 Introdução 12

I Compressão de Fontes com Perdas 17

2 Fundamentos da Compressão de Fontes 18

2.1 Teoria Taxa-Distorção 18

2.2 Cálculo da Função Taxa-Distorção 22

2.3 Quantização 26

2.4 Quantização de Taxa Fixa 30

2.5 Quantização com Restrição de Entropia 31

3 Códigos de Permutação Escalar $\quad 34$

3.1 Definição 34

3.2 Codificação Ótima $\quad 35$

3.3 Projeto de SPC 36

\begin{tabular}{lll}
\hline 3.4 & Desempenho & 39
\end{tabular}

3.5 Equivalência entre SPC e ECSQ 42

4 Códigos de Permutação Vetorial $\quad 44$

4.1 Definições $\quad 45$

4.2 Codificação Ótima 47

4.3 Equivalência entre VPC e ECVQ 48

$\begin{array}{lll}4.4 & \text { Projeto de VPC } & 51\end{array}$

4.5 Desempenho $\quad 55$

$\begin{array}{lll}4.6 & \text { Comentários } & 59\end{array}$

II Modulação $\quad 61$

5 Transmissão Digital $\quad 62$

5.1 Modelo Vetorial 62

5.2 Parâmetros de Comparação 65

5.3 Exemplos 68

6 Códigos de Permutação para Modulação $\quad 71$

\begin{tabular}{lll}
\hline 6.1 & Definição & 72
\end{tabular}

6.2 Detecção Ótima

$\begin{array}{lll}6.3 & \text { Projeto de SPM e VPM } & 75\end{array}$

6.4 Desempenho 80

6.5 Comentários 83

7 Conclusões $\quad 84$

7.1 Sugestões para trabalhos futuros $\quad 85$ 
\begin{tabular}{lr}
\hline A Algoritmo de Blahut & 88
\end{tabular}

\begin{tabular}{ll}
\hline B Estimativas de Valores Esperados & 90
\end{tabular}

C Cálculo Diferencial Matricial $\quad 92$

$\begin{array}{lr}\text { Bibliografia } & 92\end{array}$

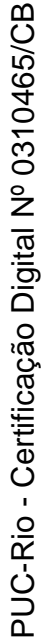




\section{Lista de Figuras}

1.1 Modelo de um sistema de comunicações. 13

2.1 Código de bloco para compressão. 19

2.2 Função densidade de probabilidade de uma fonte uniforme. 23

2.3 Função taxa-distorção para uma fonte uniforme. 23

2.4 Função densidade de probabilidade de uma fonte gaussiana. 24

2.5 Função taxa-distorção para uma fonte gaussiana. 25

2.6 Função densidade de probabilidade de uma fonte laplaciana. 25

2.7 Função taxa-distorção para uma fonte laplaciana. 26

2.8 Quantizador. 28

3.1 Comparação entre SPC's e o ECSQ ótimo para uma fonte gaussiana. O desempenho de outros quantizadores e a função taxa-distorção são também mostrados. 40

3.2 Comparação entre SPC's e o ECSQ ótimo para uma fonte laplaciana. 41

3.3 Comparação entre SPC's e o ECSQ ótimo para uma fonte uniforme. 41

3.4 Comparação entre SPC's e o ECSQ ótimo para uma fonte uniforme.

4.1 Comparação entre VPC's com $L=1,2,3$ e 10 e o ECSQ ótimo para uma fonte uniforme. $\quad 56$

4.2 Comparação entre VPC's com $L=2$ e o ECVQ para uma fonte gaussiana. O desempenho do ECSQ ótimo é também mostrado. 58

4.3 Desempenho de VPC's com $L=2$ em função do comprimento $n$. 58

4.4 Comparação entre VPC's com $L=3$ e o ECVQ para uma fonte gaussiana. O desempenho do ECSQ ótimo é também mostrado. 59

5.1 Modelo de um sistema de transmissão digital.

5.2 Modelo de um sistema de transmissão utilizando formas de onda associadas a vetores.

5.3 Modelo vetorial de um sistema de transmissão.

6.1 Exemplo de alfabeto de VPM baseado no reticulado $A_{2}$. Parâmetros: $L=2, K=19$ e $d_{\min }^{*}=1$.

6.2 Exemplo de alfabeto de VPM baseado no reticulado $D_{3}$. Parâmetros: $L=3, K=13$ e $d_{\min }^{*}=1$. $\quad 79$

6.3 Desempenho de VPM's com $L=1$ e 2 . $\quad 80$

6.4 Desempenho de VPM's com $L=2$ e $3 . \quad 81$

6.5 Desempenho de VPM's com $L=1,2$ e 3. Curvas para $n=\infty$ correspondem ao desempenho assintótico de cada código. 81

6.6 Desempenho de VPM's com $L=1,2$ e 3 para uma taxa $R=1$ em função do número total de dimensões $N=n L$. 


\section{Lista de Algoritmos}

2.1 Projeto de Quantizador MMSE $\quad 31$

2.2 Projeto de ECVQ 32

3.1 Codificação Ótima de SPC 36

3.2 Projeto de SPC 39

4.1 Projeto de Alfabeto de VPC 53

4.2 Projeto de VPC $\quad 55$

A.1 Cálculo da Função Taxa-Distorção

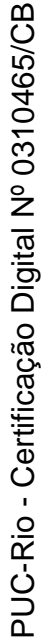

